\title{
Children's transposition as related to ratio of the training stimuli and language
}

\author{
STEPHEN F. ROBBINS and KENNETH L. WITTE \\ University of Arkansas, Fayetteville, Arkansas 72701
}

\begin{abstract}
Preschool-aged children were trained to respond to the larger of two stimuli that differed by either a 1.4:1 or a 1.96:1 ratio. The smaller, unrewarded stimulus was faded in across trials, resulting in errorless discrimination learning. After training, children were tested for transposition with stimuli either one or four steps larger than the training set. Children able to verbalize the relevant concept showed uniform relational responding, regardless of the training ratio. Included in the study were additional children unable to verbalize the relevant concept and trained with the larger ratio. A distance effect was obtained for these children.
\end{abstract}

In a 1966 study on transposition, Zeiler trained and tested 4- and 5-year-old children on stimulus pairs that had an intrapair area factor of either $1.4: 1$ or $1.96: 1$. All children were able to verbalize the relevant relationship after testing. The children trained with the 1.96:1 ratio learned the initial discrimination faster than the other children and transposed on tests that were one, two, three, and four steps removed from the training set. A distance effect was obtained for the children trained with the $1.4: 1$ ratio, that is, the amount of transposition decreased as the difference between the training and test stimuli increased. Zeiler concluded that the nature of the transposition gradient may be related to the difficulty of the training problem (learning speed), with slow learning resulting in decreasing gradients and fast learning producing flat gradients.

Osen and Cole (1967) conducted a replication and extension of Zeiler's (1966) study. These authors indicated that a confounding existed in Zeiler's study, viz., those children with a given training ratio experienced the same test ratio. Thus, Zeiler's results, rather than reflecting the effects of the ratio of the training set, may have indicated the influence of the test ratio upon transposition. Therefore, Osen and Cole used a factorial design in order to obtain a direct comparison of the separate effects of the training and test ratios (1.4:1 vs. 1.96:1) upon the transposition of nursery school children, all of whom were able to verbalize the relevant relationship. Speed of learning the training problem was found to be affected by the stimulus ratio, as children trained with the $1.96: 1$ ratio learned faster than children trained with the other ratio, thus replicating Zeiler's training results. An analysis of transposition on the first test trial indicated that the size of the test ratio did not affect transposition. With

Reprint requests should be sent to Stephen F. Robbins, who is now at the Department of Psychology, Winona State College, Winona, Minnesota 55987. respect to the effects of the training ratio, Zeiler's transposition results were replicated only to the extent that the amount of transposition for the children trained with the 1.96:1 ratio was greater than chance, while that for the other ratio group was not.

While Osen and Cole (1967) were only partially successful in replicating Zeiler's (1966) results, it is nonetheless clear that the amount of transposition is directly related to the ratio of the training stimuli, with more transposition resulting from a larger ratio $(1.96: 1)$ than from a smaller one $(1.4: 1)$. It is probable that the variable mediating the relationship between the training ratio and transposition is the degree of original learning. In both studies, all children were trained to a criterion of five successive choices of the correct stimulus. Since children trained with the larger ratio learned faster than children trained with the smaller ratio, the degree of learning should have been greater for the former group. Thus, the amount of generalization should be greater for the larger ratio group, resulting in a flatter transposition gradient.

One purpose of the present study was to determine the effects of the training ratio upon children's transposition when the degree of learning was the same for the ratio groups. All children were given the same number of training trials by means of a fading-in procedure designed to produce errorless discrimination learning. It was expected that transposition differences between the two ratio groups $(1.96: 1$ vs. $1.4: 1)$ would not be obtained, since the amount of learning was equivalent.

A second purpose of the study was to compare the transposition of children given the easy training problem (larger ratio) and either able or unable to verbalize the relevant relationship. Zeiler (1966), in discussing the effects of speed of original learning and verbalization upon transposition, hypothesized that uniform relational responding may be a function of the interaction of an easy discrimination with verbal processes. If this hypothesis is correct, then children able to verba- 
lize the relevant concept should exhibit uniform transposition, while a distance effect should be obtained for children unable to verbalize the relevant concept.

\section{METHOD}

Stimuli

The stimuli were black pasteboard squares. Two sets of stimuli were used, having area ratios $1.4: 1$ and $1.96: 1$, respectively. In the $1.4: 1 \mathrm{set}$, the areas of the training squares were 15.02 and $21.09 \mathrm{~cm}^{2}, 21.09$ and $29.47 \mathrm{~cm}^{2}$ for the one-step near test, and 57.79 and $80.81 \mathrm{~cm}^{2}$ for the fourstep far test. In the 1.96:1 set, the areas of the training squares were 15.02 and $29.47 \mathrm{~cm}^{2}, 29.47$ and $57.79 \mathrm{~cm}^{2}$ for the one-step near test, and 200.59 and $393.19 \mathrm{~cm}^{2}$ for the fourstep far test.

A series of nine squares were used to fade in the smaller square of each training pair. The first square was $2.45 \mathrm{~cm}^{2}$. The remaining eight squares of the series were formed by adding $.79 \mathrm{~cm}$ to the sides of the preceding square in the series.

\section{Procedure}

The children were randomly assigned to one of the four groups formed by the factorial combination of ratio conditions (1.4:1 vs. 1.96:1) and type of transposition test (near vs. far). All testing was done individually. The children pointed at the stimulus they thought correct, and correct choices were rewarded with small pieces of candy.

Each child was given 10 fading-in trials, followed by 10 training trials. On the first fading-in trial only the larger stimulus of the pair was presented; both stimuli were presented on all the remaining trials. Choice of the large stimulus was always correct. Following the last training trial, the experimenter pointed at the stimulus selected by the child and asked the child the reason for that choice. The child's response was recorded without comment.

The children were then given 10 transposition trials. The ratio of the test stimuli was the same as that during training. All responses were rewarded during testing. Following the last transposition test trial, the children were asked a series of questions to see if they could verbalize the concept relevant to the discrimination problems.

\section{Subjects}

The subjects were 54 children obtained from three nursery schools in Fayetteville, Arkansas. The children ranged in age from 35 to 76 months, with a mean age of 55.89 months. The data for an additional eight children, all trained with the 1.4:1 ratio, were discarded. Two of these children made more than one error during the fading-in and training trials. Of the remaining six children, all of whom were unable to verbalize the relevant concept, four were tested with the near transposition test and two were given the far test. These Ns were judged too small to make meaningful comparisons with the other six groups of the factorial design involving training ratio, type of transposition test, and ability to verbalize the relevant concept.

\section{RESULTS AND DISCUSSION}

No child made more than one error during the fading-in and training trials. The mean numbers of transposition responses, as related to training ratio, type of transposition test, and ability to verbalize the relevant concept, are presented in Table 1. These data were combined for two factorial analyses designed for unequal cell frequencies (Winer, 1962).
Table 1

Number of Transposition Responses on 10 Trials as Related to Training Ratio, Type of Transposition Test, and Verbalization Ability

\begin{tabular}{|c|c|c|c|c|c|}
\hline \multirow{3}{*}{$\begin{array}{c}\text { Train- } \\
\text { ing } \\
\text { Ratio }\end{array}$} & \multirow{3}{*}{$\begin{array}{c}\text { Verbalized } \\
\text { Relevant } \\
\text { Concept }\end{array}$} & \multicolumn{4}{|c|}{ Transposition Test } \\
\hline & & \multicolumn{2}{|c|}{ Near } & \multicolumn{2}{|c|}{ Far } \\
\hline & & $\mathrm{N}$ & Mean & $\mathrm{N}$ & Mean \\
\hline $1.4: 1$ & $\begin{array}{l}\text { Yes } \\
\text { ( No) }\end{array}$ & $\begin{array}{c}10 \\
\text { ( } 4)\end{array}$ & $\begin{array}{c}10.00 \\
\left(\begin{array}{c}6.50)\end{array}\right)\end{array}$ & $\begin{array}{l}15 \\
\text { ( } 2)\end{array}$ & $\begin{array}{l}9.87 \\
(5.00)\end{array}$ \\
\hline $1.96: 1$ & $\begin{array}{l}\text { Yes } \\
\text { No }\end{array}$ & $\begin{array}{l}8 \\
7\end{array}$ & $\begin{array}{l}8.50 \\
9.29\end{array}$ & $\begin{array}{l}7 \\
7\end{array}$ & $\begin{array}{r}10.00 \\
5.57\end{array}$ \\
\hline
\end{tabular}

Note-Data in parentheses not included in analyses.

The first set of analyses compared the data only for the 40 children able to verbalize the relevant concept. A preliminary analysis indicated that the ages of the children in the four groups, training ratio by type of transposition test, did not differ reliably. The analysis of transposition scores indicated that neither the main effect nor the interaction was significant. Thus, as predicted, transposition differences between the two ratio groups were not obtained. Therefore, degree of learning, rather than ease of learning, would seem to be the critical variable responsible for the differential transposition gradients obtained in the earlier investigations of the effects of training ratios upon transposition (Osen \& Cole, 1967; Zeiler, 1966).

The second set of analyses compared the data for the 29 children trained with the $1.96: 1$ ratio. An initial factorial analysis, ability to verbalize the relevant concept by type of transposition test, of the ages of the children in the four groups resulted in no reliable differences. The analysis of the transposition scores indicated that both main effects were nonsignificant $[F(1,25)=5.52]$. As is apparent from an inspection of Table 1, a distance effect was obtained for the children unable to verbalize the relevant concept, while uniform relational responding was obtained for the other children. Furthermore, the nature of the interaction is consistent with Zeiler's (1966) hypothesis that uniform relational responding may depend upon the interaction of verbal processes and ease of learning (degree of learning).

The present results, in addition to confirming the two experimental hypotheses, also replicate and extend earlier results obtained by Cole, Dant, Eguchi, Fujii, and Johnson (1964). These authors used the fading-in training procedure as a means of testing Spence's (1937, 1942) theory of transposition. Since this procedure results in no training errors, no conditioned inhibition should develop. As a consequence, subjects should exhibit uniform absolute responding when tested for transposition. Cole et al. found that preschool children, all unable to verbalize the relevant concept and all trained and tested with stimulus pairs having a $1.6: 1$ ratio, exhibited uniform relational responding on a 
near test rather than the absolute responding predicted by Spence. Cole et al., rather than criticizing Spence's theory, suggested that human subjects can establish and maintain an inhibitory set with little or no experience involving responding to the unrewarded training stimulus. Regardless of the validity of their analysis, on the basis of their findings and the present results it is apparent that the effects of the fading-in training procedure upon the transposition of children unable to verbalize the relevant concept are the same as those obtained in studies (e.g., Kuenne, 1946) in which children responded to the incorrect stimulus and experienced nonreinforcement, viz., a distance effect is obtained.

\section{REFERENCES}

Cole, R. E., Dant, H. E., Eguchi, P. E., Fuji, K. K., \& Johnson, R. C. Transposition with minimal errors during training trials. Journal of Experimental Child Psychology, 1964, 1, 355-359.

KuENNE, M. R. Experimental investigation of the relation of language to transposition behavior in young children. Journal of Experimental Psychology, 1946, 36, 471-490.

Osen, L., \& Cole, M. Transposition as a function of withinpair differences. Psychonomic Science, 1967, 8, 545-546.

SPENCE, K. W. The differential response in animals to stimuli varying within a single dimension. Psychological Review, 1937, 44, 430-444.

SPENCE, K. W. The basis of solution by chimpanzees of the intermediate-size problem. Journal of Experimental Psychology, 1942, 31, 257-271.

WINER, B. J. Statistical principles in experimental design. New York: McGraw-Hill, 1962.

ZeILER, M. D. Solution of the two-stimulus transposition problem by four- and five-year-old children. Journal of Experimental Psychology, 1966, 71, 576-579.

\author{
(Received for publication January 20, 1978.)
}

